\title{
Inferring directed networks using a rank-based connectivity measure
}

\author{
Marc G. Leguia ${ }^{1,2},{ }^{*}$ Cristina G. B. Martínez ${ }^{2}$, Irene Malvestio ${ }^{2,3,4}$, Adrià Tauste \\ Campo $^{5,6,7}$, Rodrigo Rocamora ${ }^{6,8}$, Zoran Levnajić ${ }^{1,9}$, and Ralph G. Andrzejak ${ }^{2,10}$ \\ Faculty of Information Studies, 8000 Novo Mesto, Slovenia ${ }^{1}$ \\ Department of Communication and Information Technologies, \\ Universitat Pompeu Fabra, 08018 Barcelona, Spain ${ }^{2}$ \\ Department of Physics and Astronomy, University of Florence, 50119 Sesto Fiorentino, Italy ${ }^{3}$ \\ Institute for Complex Systems, CNR, 50119 Sesto Fiorentino, Italy ${ }^{4}$ \\ Center for Brain and Cognition, Department of Information and Communication Technologies, \\ Universitat Pompeu Fabra, 08018 Barcelona, Spain ${ }^{5}$ \\ Epilepsy Unit, Department of Neurology, IMIM Hospital del Mar, \\ Universitat Pompeu Fabra, 08003 Barcelona, Spain ${ }^{6}$ \\ Barcelonaßeta Brain Research Center, Pasqual Maragall Foundation, 08005 Barcelona, Spain ${ }^{7}$ \\ Faculty of Health and Life Sciences, Universitat Pompeu Fabra, 08003 Barcelona, Spain ${ }^{8}$ \\ Institute Jozef Stefan, 1000 Ljubljana, Slovenia ${ }^{9}$ and \\ Institute for Bioengineering of Catalonia (IBEC), \\ The Barcelona Institute of Science and Technology, Baldiri Reixac 10-12, 08028 Barcelona Spain ${ }^{10}$
}

(Dated: June 14, 2019)

\begin{abstract}
Inferring the topology of a network using the knowledge of the signals of each of the interacting units is key to understanding real-world systems. One way to address this problem is using datadriven methods like cross-correlation or mutual information. However, these measures lack the ability to distinguish the direction of coupling. Here, we use a rank-based nonlinear interdependence measure originally developed for pairs of signals. This measure not only allows one to measure the strength but also the direction of the coupling. Our results for a system of coupled Lorenz dynamics show that we are able to consistently infer the underlying network for a subrange of the coupling strength and link density. Furthermore, we report that the addition of dynamical noise can benefit the reconstruction. Finally, we show an application to multichannel electroencephalographic recordings from an epilepsy patient.
\end{abstract}

\section{INTRODUCTION}

A rich variety of natural and man-made systems are composed of separate components that interact in a complex way. These systems are modeled by using networks where each component is represented by a node and their interaction is conveyed by links between the nodes. This approach helped to advance our understanding of realworld systems ranging from social to natural sciences [1]. To understand the collective dynamics of these complex systems, it is essential to characterize not only the behavior of individual nodes, but also the network structure that makes them interact via links.

For many real-world systems, while the network structure is not directly accessible, one can measure signals from each of the nodes. Therefore, it is important to develop methods for inferring the topology from measured signals. Research that aims at this inference can be found in the context of biology [2], neurobiology [3, 4] and climate science [5], among others.

In general, we can address the inference problem in two different ways depending on the type of assumptions we make. One approach is to use certain models for the nodes and possibly also for the type of interaction. In combination with the temporal evolution of the dynam-

*mgrauleg@gmail.com ics of each node, this information allows one to reconstruct the network. In some cases, the parameters of the model can also be determined [6-14]. These type of methods can only work when the assumptions about the model are correct. The second approach to address the inference problem is to use exclusively the knowledge of the temporal evolution without selecting any model. Although data-driven approaches make implicit assumptions of the measured signals, for example whether the dynamics are linear or nonlinear, it remains more applicable to real-world systems. Using this approach, measures like cross correlation, mutual information, and mutual information rate can perform well [15-17]. However, these measures are not able to determine the direction of interaction between nodes. Partial mutual information from mixed embedding is able to infer networks which have almost the same topological structures like the original ones $[18,19]$. Furthermore, measures like partial directed coherence and partial transfer entropy were used for small systems with particular topologies [20-24].

Despite the progress summarized above, inferring the exact connectivity matrix for larger directed networks without a specific choice of the topology remains a difficult task. In this paper, we address this task using a data-driven approach. For this purpose, we use the state space measure $L$ defined in Ref. [25]. This measure belongs to a family of measures which aim to detect the direction of couplings between dynamics from pairs of signals by using the asymmetric state similarity crite- 
rion [26-32]. Applications of $L$ to real-world data include electroencephalographic (EEG) recordings from epilepsy patients [33, 34] and neurophysiological signals [35]. In Ref. [36] the efficacy of $L$ in the detection of bidirectional couplings was studied, and in Refs. [31, 37] $L$ was used to detect coupling from spike train dynamics. Here, we apply $L$ to multivariate signals extracted from networks by analyzing all pairwise combinations of individual signals, resulting in estimates of the connectivity matrix.

We study the performance of our method using Lorenz oscillators as the dynamics for each node arranged in a random network with diffusive couplings between nodes. The influence of parameters like the coupling strength, and the link density is illustrated. In particular, we show that for a range of these parameters, we are able to infer perfectly the underlying network of the system. We furthermore show that the addition of dynamical noise can in some cases improve the performance of the method. Moreover, we study the robustness of the method for increased system sizes. Finally, we report an application of our method to EEG recordings of seven seizures from an epilepsy patient. We illustrate the temporal evolution of the connectivity prior, during, and after the seizure.

\section{METHODS}

In the following we describe the measure $L$ [25] which is based on the asymmetric state similarity criterion. Assume that we have two dynamical systems $X$ and $Y$ from which we derive scalar signals $x_{i}$ and $y_{i}$ for $i=1, \ldots, N^{*}$. From these signals, we reconstruct the state space of the dynamics using delay vectors $\mathbf{x}_{i}=$ $\left(x_{i}, x_{i-\tau}, \ldots, x_{i-(m-1) \tau}\right), \quad \mathbf{y}_{i}=\left(y_{i}, y_{i-\tau}, \ldots, y_{i-(m-1) \tau}\right)$ where $m$ is the embedding dimension and $\tau$ is the time delay. The index $i$ now goes from $i=i_{o}=1, \ldots, N=$ $N^{*}-(m-1) \tau$ so the delay vector with index $i$ has the scalar value with that same index $i$ as a leading element. For $j=1, \ldots, k$, we denote by $v_{i, j}$ and $w_{i, j}$ the time indices of the $k$ spatially nearest neighbors of $\mathbf{x}_{i}$ and $\mathbf{y}_{i}$, respectively.. Here the Euclidean distance is used as metric, and temporally nearest neighbors are discarded by applying a Theiler window of length $T$ [38]. Accordingly, the time indices must satisfy that $\left|v_{i, j}-i\right|>T$ and $\left|w_{i, j}-i\right|>T$. We sort the distances between $\mathbf{x}_{i}$ and $\mathbf{x}_{i_{o} \neq i}$, and we introduce $g_{i, i_{o}}$ which stands for the rank of the distance between $\mathbf{x}_{i}$ and $\mathbf{x}_{i_{o}}$ in an ascending list. To quantify the interdependence from $X \rightarrow Y$ we use $L(X \mid Y)$ defined in the following way. We take the $X \mathrm{dy}-$ namics as reference, and define the $Y$-conditioned mean rank as: $G_{i}^{k}(X \mid Y)=\frac{1}{k} \sum_{j=1}^{k} g_{i, w_{i, j}}$. We then introduce:

$$
L(X \mid Y)=\frac{1}{N} \sum_{i=1}^{N} \frac{G_{i}(X)-G_{i}^{k}(X \mid Y)}{G_{i}(X)-G_{i}^{k}(X)},
$$

where $G_{i}(X)=\frac{N}{2}$ and $G_{i}^{k}(X)=\frac{k+1}{2}$ correspond to the mean and minimal mean rank of the distances in the $X$ signal, respectively. To compute $L(Y \mid X)$, which quantifies the interdependence from $Y \rightarrow X$, we just exchange the roles of $X$ and $Y$. For identically synchronized dynamics $(X=Y)$, we get $L(X \mid Y)=1$. In contrast, for independent dynamics the expected value of $L(Y \mid X)$ is distributed around zero. The number of nearest neighbors is set to $k=5$, and we use $m=5$ and $\tau=5$ for the embedding dimension and the time delay, respectively. Finally, for the Theiler window we use $T=15$. These values are taken from Ref. [36] to avoid any in-sample parameter optimization.

We apply our method to directed networks with a dynamical system at each of the $M$ network nodes. We use random networks, which are defined by the link probability $\rho$. The existence of a directed link between two nodes is given by this probability $\rho$. By construction, we exclude self-loops, i.e. nodes are never connected with themselves. Links between nodes are expressed by the binary adjacency matrix $A$. More specifically, the matrix element $A_{p q}=1$, if a directed link from $p$ to $q$ exists. The equality $A_{p q}=A_{q p}=1$ holds, if the link is bidirectional. In contrast, $A_{p q}=0$ if there is no link from $p$ to $q$. The range of the matrix indices is $p, q \in(1, . ., M)$. Since we use directed networks, in general $A_{p q} \neq A_{q p}$. Our aim in this paper, is to infer the matrix $A$ using only the information of the scalar signals derived from each of the $M$ nodes.

We consider directed networks of size $M=16$ with Lorenz dynamics at each node. The nodes are connected via a diffusive coupling with strength $\varepsilon$ through the $x$ components:

$$
\begin{aligned}
& \dot{x_{p}}(t)=10\left(y_{p}(t)-x_{p}(t)\right)+\varepsilon \sum_{q=1}^{M} A_{q p}\left[x_{q}(t)-x_{p}(t)\right]+\xi_{p}^{x}(t), \\
& \dot{y_{p}}(t)=x_{p}(t)\left[b_{p}-z_{p}(t)\right]-y_{p}(t)+\xi_{p}^{y}(t), \\
& \dot{z_{p}}(t)=x_{p}(t) y_{p}(t)-\frac{8}{3} z_{p}(t)+\xi_{p}^{z}(t) .
\end{aligned}
$$

We study two different settings. A heterogeneous system by taking $b_{p}$ from a uniform distribution in the interval $[28,48]$ or a homogeneous system by setting $b_{p}$ to a constant value of 28 . In this setting, the model is chaotic at zero coupling strength. The quantity $\xi(t)$ stands for independent Gaussian noise with zero mean and with the following correlation function $<\xi_{p}^{l}(t) \xi_{q}^{l^{\prime}}\left(t^{\prime}\right)>=$ $2 D \delta_{l l^{\prime}} \delta_{p q} \delta\left(t-t^{\prime}\right)$ with $l$ and $l^{\prime}$ being the three components of the Lorenz dynamics. For a given link probability $\rho$ and coupling strength $\varepsilon$, we draw $S=10$ independent random realizations of the adjacency matrix $A$. For each of these realizations of the network structure, we generate $R=200$ realizations of the dynamics. For each realization of the dynamics, we start the system at random initial conditions and use a fourth-order Runge-Kutta scheme to integrate the dynamics. We use an integration step of 0.05 time units and downsample by a factor of 6 . We discard initial transients, and extract the $x$ component of the Lorenz dynamics for each 
of the network nodes. We use 4096 data points, which corresponds to approximately 200 oscillation periods.

For a given adjacency matrix $A$ and each of the $R=$ 200 realizations of the dynamics, we compute the matrix elements $W_{p q}=L\left(X_{p} \mid X_{q}\right)$. This matrix contains the $L$ values for all the possible pairs of signals in the network. In general, the values $L\left(X_{p} \mid X_{q}\right)$ and $L\left(X_{q} \mid X_{p}\right)$ are not equal, and we therefore get an asymmetric matrix. We skip the diagonal elements which correspond to $L\left(X_{p} \mid X_{p}\right)=1$. We average $W$ across the $R=200$ dynamical realizations to obtain $\bar{W}$. Our objective is to reconstruct the true underlying topology $A$ by separating the continuous valued entries of the matrix $\bar{W}$ into two sets. To achieve this separation, we apply a threshold $\eta$ to obtain a reconstructed binary adjacency matrix $R^{\eta}$ :

$$
R_{p q}^{\eta}= \begin{cases}0 & \text { if } \bar{W}_{p q} \leq \eta \\ 1 & \text { if } \bar{W}_{p q}>\eta\end{cases}
$$

with $p, q \in(1, . ., M)$ without considering diagonal elements $p=q$.

To quantify the accuracy with which $R^{\eta}$ estimates the true connectivity $A$, we compute the true positive rate $\lambda^{\eta}$ which is defined by the number of correctly detected links divided by the total number links:

$$
\lambda^{\eta}=\frac{\sum_{p, q ; p \neq q}^{M} R_{p q}^{\eta} \cdot A_{p q}}{\sum_{p, q ; p \neq q}^{M} A_{p q}} .
$$

The false positive rate $\kappa^{\eta}$ is defined by the number of incorrectly detected links divided by the total number of absent links:

$$
\kappa^{\eta}=\frac{\sum_{p, q ; p \neq q}^{M} R_{p q}^{\eta} \cdot B_{p q}}{\sum_{p, q ; p \neq q}^{M} B_{p q}}, \text { with } B_{p q}= \begin{cases}0 & \text { if } A_{p q}=1 \\ 1 & \text { if } A_{p q}=0\end{cases}
$$

We then compute the reconstructed matrices $R^{\eta}$ in dependence on the threshold $\eta$ and determine the resulting values of $\lambda^{\eta}$ and $\kappa^{\eta}$. By plotting $\lambda^{\eta}$ versus $\kappa^{\eta}$ for all values of $\eta$ we get the so-called receiver operating characteristic (ROC) curve. We use the area under the ROC curve to quantify the accuracy of the reconstruction. This area, which we denote by $\Omega$, attains its maximal value of one for a perfect classification. In this case, a threshold $\eta$ exists for which we get $R^{\eta}=A$. In contrast, the expected value for a random classification of this area is $\Omega=0.5$.

\section{RESULTS}

We start by analyzing the elements of the matrix $\bar{W}$ for four exemplary cases [Fig. 1(a)-1(d)]. All examples are computed from the heterogeneous noise-free system
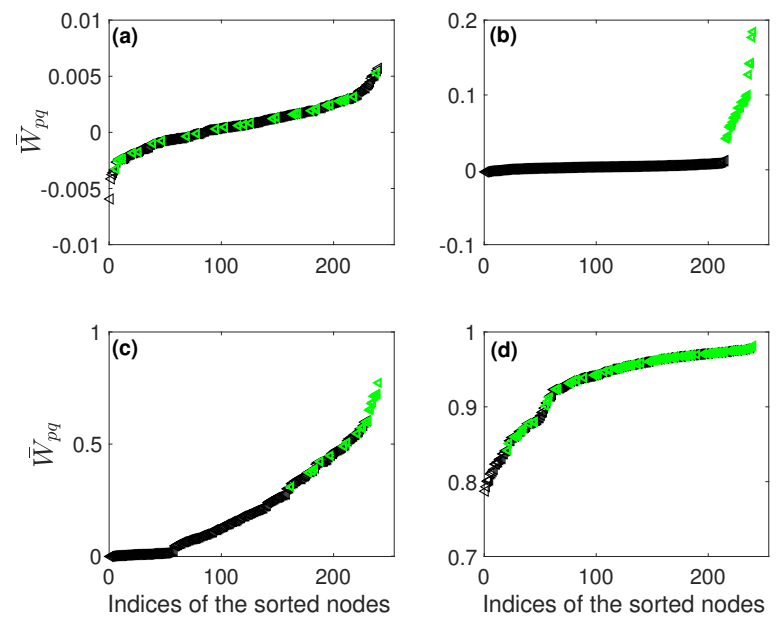

FIG. 1. Different coupling strengths and link densities lead to different qualities of the network reconstruction. Sorted $\bar{W}$ values for four different exemplary cases (a-d) computed for the heterogeneous noise-free case. For the cases (a-c), we fix $\rho=0.1$ with $\varepsilon=0,0.665$, and 4.92 , respectively. For the case (d), we use $\rho=0.4$ and $\varepsilon=4.92$. Values corresponding to links (no links) are marked in green (black).

of Lorenz oscillators. In Fig. 1(a)-1(c), we use the coupling strengths $\varepsilon=0,0.665$, and 4.92 , respectively, while the link density is set to $\rho=0.1$. In Fig. 1(d), we use $\varepsilon=4.92$ but with higher link density $\rho=0.4$. In order to evaluate $\bar{W}$, we plot its elements $\bar{W}_{p q}$ in an ascending order [15], in green if there is a link $\left(A_{p q}=1\right)$ and black otherwise $\left(A_{p q}=0\right)$. For zero coupling [Fig. 1(a)], all $\bar{W}_{p q}$ values are around zero and there is no difference between the values where $A_{p q}=1$ and $A_{p q}=0$. In contrast, for intermediate coupling [Fig. 1(b)], there is a clear gap between results obtained from pairs of signals from connected nodes on the one hand, and results obtained from unconnected nodes on the other hand. Any threshold $\eta$ taken from within this gap will lead to a perfect reconstruction of the adjacency matrix: $R^{\eta}=A$. For high coupling [Fig. 1(c)], the $\bar{W}_{p q}$ generally increase. Due to this increase, values obtained from pairs of signals from connected nodes and values obtained from unconnected nodes mix. Therefore, the gap we had in Fig. 1(b) closes. Nonetheless, results for connected nodes still tend to be higher than results for unconnected nodes. Finally, in Fig. 1(d), the increased link density paired with the high coupling leads to an almost synchronous temporal evolution for all nodes. As a result, the elements of $\bar{W}_{p q}$ are close to the upper bound of the underlying measure $L$ which is 1 . From this display of the results in Fig. 1(d), we still see a tendency of connected nodes to be at higher values, but a clear distinction between the values from connected and unconnected nodes is no longer possible. For cases where $A$ is not know, an analysis of the the ordered set of $\bar{W}$ could be also performed in order to help with the threshold decision. 


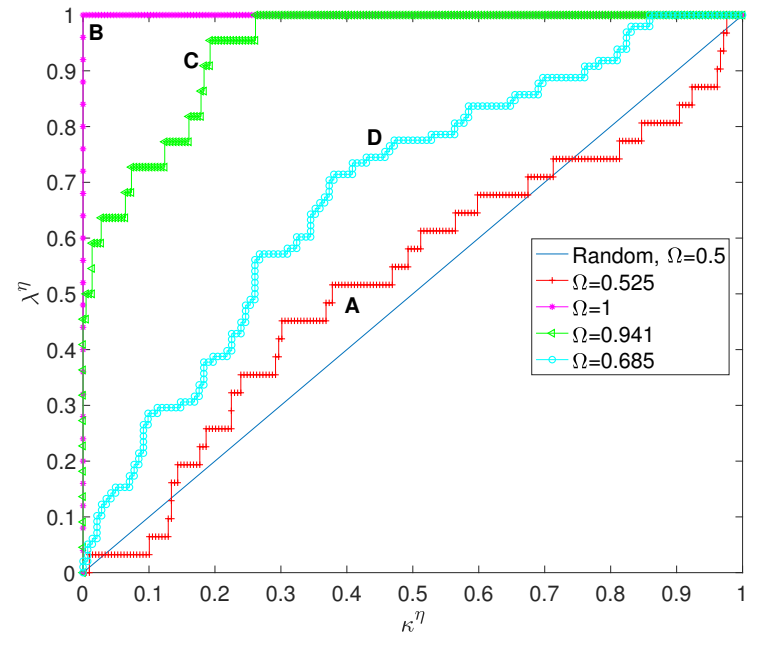

FIG. 2. ROC curves allow us to quantify the accuracy of the reconstruction for a variable threshold. ROC curves for a heterogeneous noise-free system using different values of $\rho$ and $\varepsilon$. The curves A-D correspond to the cases (a-d) in Fig. 1. The blue line corresponds to the ROC curve of a random classification. In the legend we show the area under the ROC curve $\Omega$.

Fig. 1(b) shows that at intermediate coupling and low link density, a gap between the sorted $\bar{W}$ values from connected and unconnected nodes appears. Any threshold $\eta$ placed within this gap, allows us to reconstruct the underlying network perfectly. In contrast, in Figs. $1(\mathrm{a}), 1(\mathrm{c})$ and $1(\mathrm{~d})$, no threshold leads to a perfect reconstruction, and there is no single optimal threshold. To further differentiate these cases, we show in Fig. 2 the ROC statistics in order to quantify the classification performance for these different cases [Fig. 1(a)-1(d)]. The resulting ROC curves (A-D) and corresponding areas under ROC curves $\Omega$, complement the information shown in Fig. 1. At zero coupling (curve A), the ROC curve has a similar shape and area $(\Omega=0.525)$ as expected for a random classification $(\Omega=0.5)$. This correctly reflects that for zero coupling there is no difference between connected and unconnected nodes. For the curve B, we have a perfect ROC curve with $\Omega=1$. This means that upon increasing the threshold we first find all the links $\lambda^{\eta}=1$. Only when increasing the threshold further we would find false positives, i.e. classify pairs of unconnected nodes as links. At high coupling strength and link density $\rho=0.1$ (curve $\mathrm{C}$ ), we find some false positives before finding all the links. This reflects the results displayed in Fig. 1(c) where the values of connected and unconnected nodes mix. Still, the classification is very high as shown by $\Omega=0.941$. Finally, for the curve D, the area drops substantially to $\Omega=0.685$ but remains higher than the random case. The fact that the classification performance remains better than random indicates that for the curve $\mathrm{D}$, although all the points had very similar values in Fig. $1(\mathrm{~d})$, the results for connected nodes have a tendency to be higher than the ones for unconnected nodes.

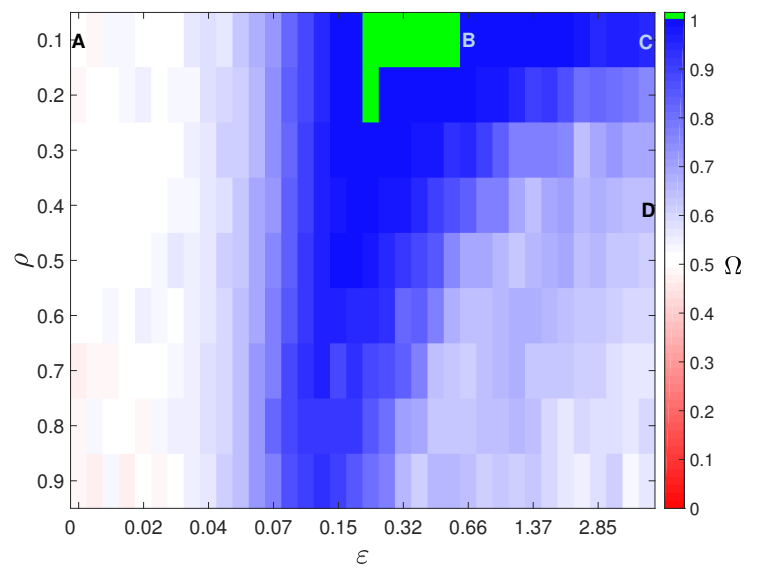

FIG. 3. Performance of the network reconstruction for the noise-free heterogeneous dynamics is highest for sparse networks with intermediate coupling between nodes. Area under the ROC curve $\Omega$ averaged across $S=10$ independent realizations of the adjacency matrix $A$ in dependence on the link probability $\rho$ and the coupling strength $\varepsilon$. Values of $\varepsilon$ are placed equidistantly on a logarithmic scale. Only when $\Omega=1$ across all the $S$ realizations we plot the corresponding field in green. Labels from A to D mark the set of parameters $(\rho, \varepsilon)$ used in both Figs. 1 and 2 .

Above we showed detailed results for individual realizations of the heterogeneous noise-free system at exemplary values of the link density $\rho$ and the coupling strength $\varepsilon$. We now study the performance of our method for ranges of these parameters, and averaged over independent realizations of the adjacency matrix $A$ (Fig. 3). For zero and small coupling strengths, the reconstruction is similar to a random classification $(\Omega \approx 0.5)$, for all $\rho$. At intermediate couplings $(\varepsilon \approx 0.07)$, the performance quickly improves, and for low $\rho$ we even have a region with perfect reconstructions $\Omega=1$ for all $S$ realizations (green area in Fig. 3). This region is surrounded by an area of high performance $\Omega \approx 0.9$ (dark blue area in Fig. 3). For small $\rho$ this region of high performance reaches high coupling values. However, as $\rho$ gets bigger, the broadness of this area reduces. For higher link densities and higher couplings the dynamics of the nodes becomes almost synchronous. It is known that a reliable extraction of directional couplings from almost synchronous dynamics is not possible $[25,39,40]$. For this reason the performance is approaching the chance level when both the link density and coupling strengths are high.

The system that we have analyzed so far is heterogeneous in the sense that the Lorenz oscillators across network nodes are non-identical in their parameter $b_{q}$. We continue to study a noise-free but now homogeneous network by setting $b_{q}=28$ for all nodes. The area under the ROC curve $\Omega$ averaged across all the $S$ resets of this homogeneous system is displayed in Fig. 4. The green region with perfect reconstruction is smaller than 


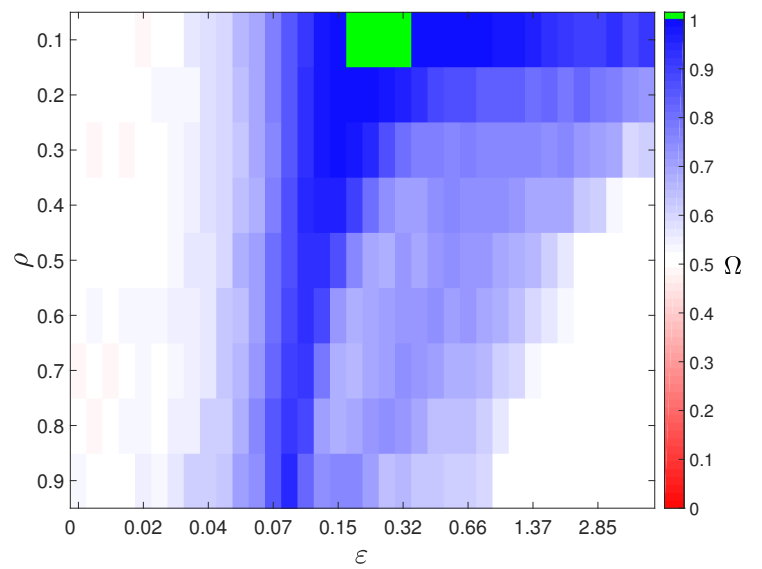

FIG. 4. Compared to the results for the heterogeneous noisefree system, the performance of the network reconstruction is degraded for the homogeneous noise-free system. Same as Fig. 3 but here for the homogeneous noise-free case.

the corresponding region in Fig. 3. Moreover, the dark blue area of high performance $(\Omega \approx 0.9)$ is also narrower than the one in the heterogeneous system. For high $\varepsilon$ and $\rho$ we find an area with exactly $\Omega=0.5$. In this region, the nodes are identically synchronized and therefore we find that $L\left(X_{p} \mid X_{q}\right)=1$ for all the $p, q$. While for thresholds $\eta>1$, we obtain $\lambda^{\eta}, \kappa^{\eta}=0$, we obtain $\lambda^{\eta}, \kappa^{\eta}=1$ for $\eta \leq 1$. Hence, the ROC curve for this configuration coincides with the diagonal, resulting in $\Omega=0.5$ which reflects that it is impossible to reconstruct a network when all nodes behave the same.

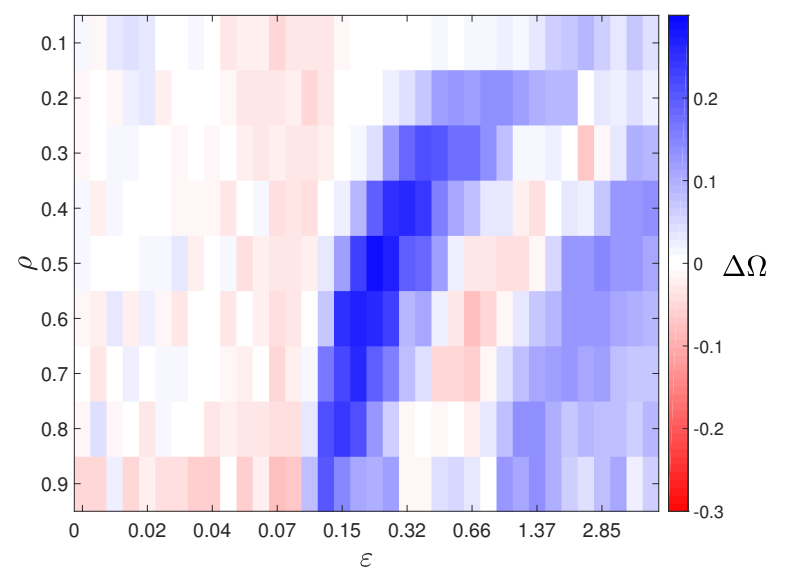

FIG. 5. For intermediate and high coupling strengths, heterogeneity helps the reconstruction. Difference in performance between the heterogeneous and the homogeneous systems $\Delta \Omega$ shown in Figs. 3 and 4 . Blue and red correspond to higher performance obtained for the heterogeneous and homogeneous system, respectively.

To further compare the results of our approach ob- tained for heterogeneous and homogeneous systems we inspect the differences between their reconstruction performances $\Delta \Omega$ (Fig. 5). For low couplings $\varepsilon \leq 0.07$, the method performs better for the homogeneous system. This shows that small couplings have stronger effects between identical dynamics as compared to non-identical dynamics. As a result, our method can detect them better. In contrast, when the coupling strength is increased, heterogeneity facilitates the reconstruction. This results in an area of positive $\Delta \Omega$ (dark blue) in the center of the parameter space of $\varepsilon$ and $\rho$ depicted in Fig. 5. Here, the coupling strength is high enough for the homogeneous system to substantially increase the overall interdependence between signals, making it more difficult to distinguish real links from indirect connections. In contrast, for the heterogeneous case, we need higher coupling to find this effect. This results in a dark blue area, indicating that the method performs much better for heterogeneous system than for the homogeneous one. For parameters located right from the central dark blue area, some substantial changes of our dynamics have to be mentioned for the interpretation of our results. For some realizations of the adjacency matrix and some initial conditions, the dynamics of a subset of the nodes degenerates from the original complex two-wing Lorenz attractor to much simpler structures and even fixed point solutions. The presence of such degenerated solutions has a negative impact on the average performance. This effect takes place at different regions of the parameter space for the homogeneous versus the heterogeneous system. This disparity is the main reason why right from the central dark blue region we find a red region for which the homogeneous system results in a better performance. Finally, for high couplings and high link density, no degeneration takes place. The performance is again better in the heterogeneous system leading to a triangular shaped blue area in the lower-right of Fig. 5. This again is because homogeneous systems are easier to synchronize than heterogeneous ones, and in consequence, it is more difficult to infer connectivity in the former type of systems.

So far we analyzed the system in Eq. 2 keeping the noise level at zero. We now study the homogeneous system with a variable degree of dynamical noise. The averaged performance $\Omega$ as a function of the noise for three different characteristic $\varepsilon$ keeping $\rho=0.5$ is plotted in Fig. 6. At low coupling strength $(\varepsilon=0.017)$, the addition of noise does not change the performance, and we have a random classification for all values of the noise. For $\varepsilon=0.107$ the performance is high for the noise-free case and decreases with increasing noise level. If we further increase the coupling strength $(\varepsilon=0.266)$, the increased coupling makes the dynamics of each node more alike and the overall interdependence in the dynamics is increased. Here, moderate noise reduces this dynamical interdependence of the signals and therefore, it is easier to infer the connectivity of the system. This effect is strongest for an optimal value of the noise $\sqrt{2 D} \approx 0.38$ for which the performance $\Omega$ achieves its maximum value. When the noise 


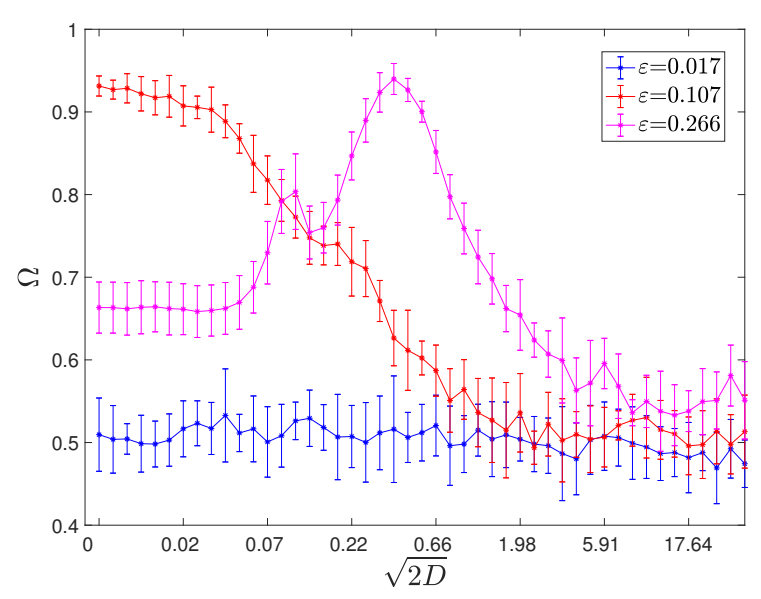

FIG. 6. Depending of the choice of $\varepsilon$ and $\rho$, there exists an ideal level of noise which improves the reconstruction as compared to the homogeneous noise-free case. Average performance of the reconstruction for $S=10$ adjacency matrix realizations as a function of the noise level for some characteristic values of $\varepsilon$ keeping $\rho=0.5$. The error bars represent the standard deviation across the ten different realizations.

is increased further, the performance approaches the one of a random classification.

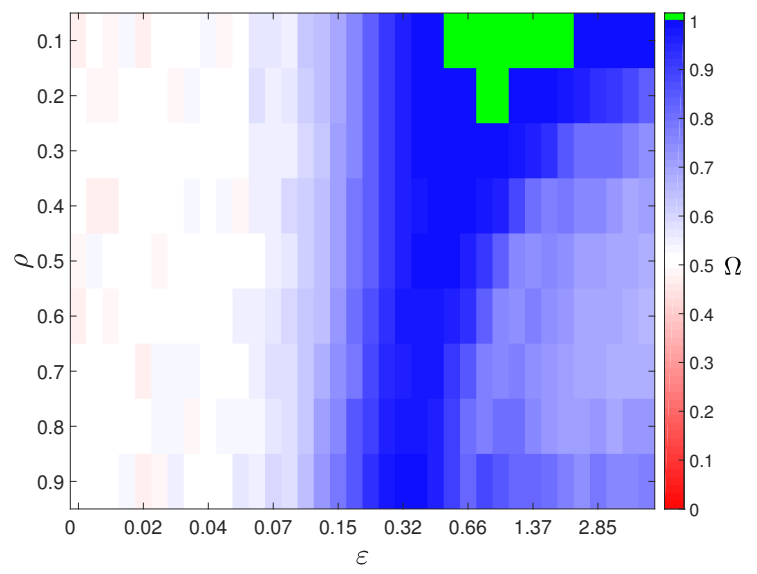

FIG. 7. Dynamical noise increases the performance for a wide range of parameters. Same as Fig. 3 but here for the homogeneous case with noise level of $\sqrt{2 D}=0.5$.

As shown in Fig. 6, the noise level leading to the highest performance depends on the parameter $\varepsilon$. In Fig. 7, we show the averaged performance of a fixed noise level of $\sqrt{2 D}=0.5$ in dependence on $\varepsilon$ and $\rho$. For small coupling strengths, we still perform like a random classification. As the coupling strength increases $(\varepsilon \approx 0.07)$, the noise hinders our approach to detect the connectivity and we continue to perform almost at random while in the noise-free case we have good performance at these parameter values. In contrast, once the coupling is high enough $(\varepsilon \approx 0.15)$, dynamical noise results in increased $\Omega$ values and we find larger regions with perfect reconstruction (green) and high performance (dark blue) than for the noise-free case. Moreover, at very high coupling we perform better than chance level in a parameter region where the classification for the noise-free case is completely random.

Finally, we study the performance of our method for increased values of the network size $M$ keeping the length of the signal and the number of dynamical realizations $R$ fixed. We study the noise-free heterogeneous case keeping $\rho=0.1$ and for four different values of the coupling strength $\varepsilon$. The averaged performance for different network sizes $M$, is shown in Fig. 8. Due to the computational cost at increased network sizes, we limit the adjacency matrix realizations to $S=5$. For sizes $M \leq 32$, the performance barely changes with the increased size. We notice that at $M=32$ we do not perform perfectly for all the realizations but we still have a high $\Omega$ across the different coupling strengths. At $M=64$, we still have very good performance for almost all the coupling strengths considered. However, the performance decreases and approaches chance level upon further increasement of $M$. This is specially true for the higher coupling strengths. To interpret this deterioration of the performance, one has to keep in mind that the number of possible links grows quadratically with the size $M$. This way, although we keep $\rho=0.1$, the average number of links at any given node is much larger at $M=256$ than at $M=16$, which in turn makes the system more synchronizable. However, most real-world networks tend to be sparse [1]. So as the system size grows, the quantity that is conserved should be the link per node and not the density. To further illustrate this point, we take as a reference quantities $\left(M_{0}=16, \rho_{0}=0.1\right)$ and define:

$$
\rho_{M}=\rho_{0} \frac{M_{0}-1}{M-1},
$$

where $\rho_{M}$ is link density at size $M$. The measure $\rho_{M}$ preserves the average links per node that we had at the one of the reference point $\left(M_{0}=16, \rho_{0}=0.1\right)$. Similar to Fig. 8, in Fig. 9 we show the average performance for increased system sizes for some characteristic $\varepsilon$ 's but using $\rho_{M}$ for the link density. For all the $\varepsilon$, we observe that the performance does not change substantially with increased system size. At $\varepsilon=0.27,0.55$, the only difference as the system size increases is that we do not perform perfectly for all the realizations computed. Still the average performance in these areas are almost perfect $\Omega>0.99$. These results confirm that the decrease in performance we observe in Fig. 8 is due to this increase of links which also make the system easier to synchronize.

\section{APPLICATION TO EEG DATA}

We now show an application of our method to EEG recordings from an epilepsy patient (patient 1 in $[41,42]$ ). 


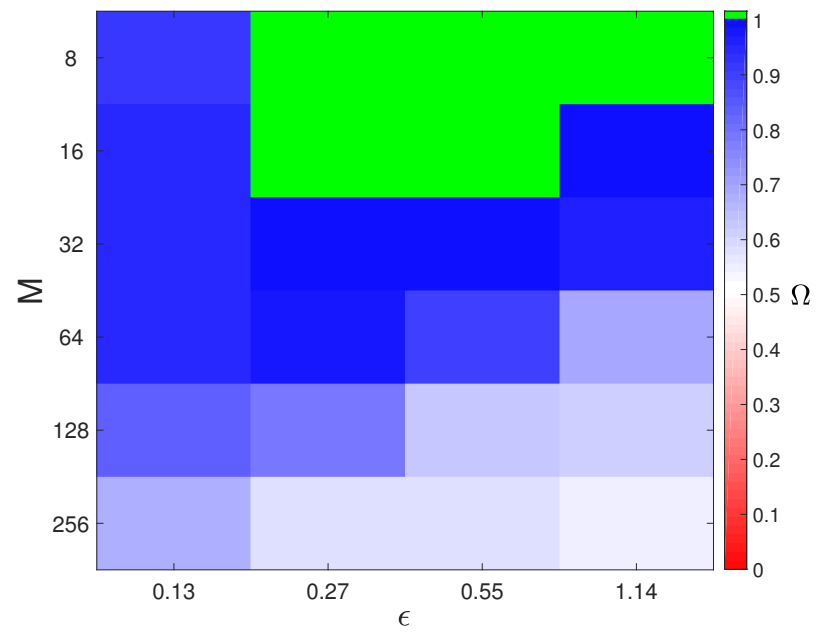

FIG. 8. Performance of the network reconstruction is decreased for increasing size $M$ when the link density $\rho$ is not adjusted. Area under the ROC curve $\Omega$ averaged across $S=5$ independent realizations of the adjacency matrix $A$ in dependence on the network size $M$ and the coupling strength $\varepsilon$. Values of $\varepsilon$ are placed equidistantly on a logarithmic scale. We use a density $\rho=0.1$ for all the cases. Only when $\Omega=1$ across all the $S$ realizations we plot the corresponding field in green.

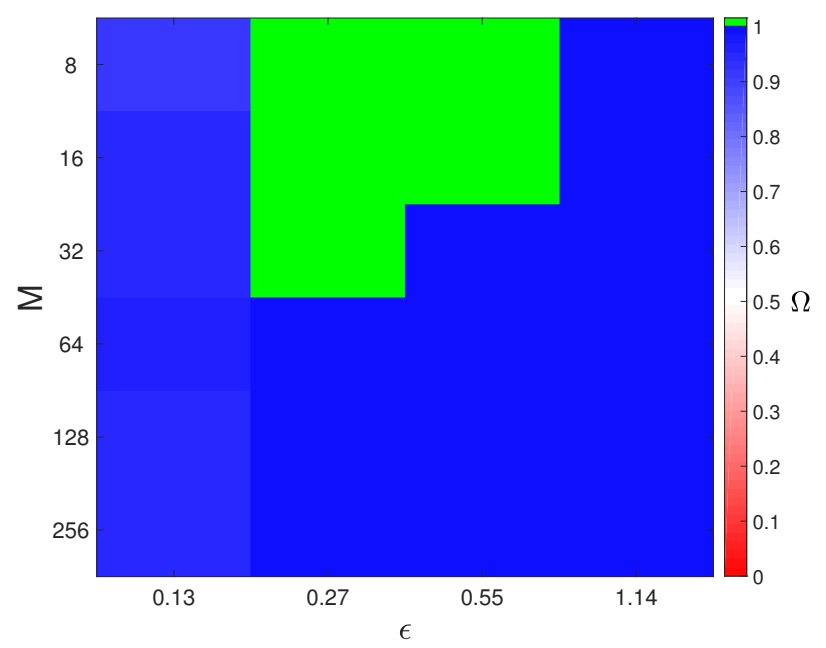

FIG. 9. Performance of the network reconstruction for the noise-free heterogeneous dynamics does not change if the system size grows. Same as Fig. 8 but using $\rho_{M}$.

These recordings were performed prior to and independently from our study in the context of this patient's pre-surgical diagnostics in Hospital del Mar, Barcelona, Spain. Five electrodes with a total of 56 recording channels were implanted intracranially in the following brain regions: frontal (FR), amygdala (AM), anterior hippocampus $(\mathrm{AH})$, posterior hippocampus $(\mathrm{PH})$ and temporo-basal cortex (TB). The patient was diagnosed with a right-sided nonlesional mesial temporal lobe epilepsy and underwent epilepsy surgery resulting in complete seizure freedom with a follow-up of more than five years.

Our dataset contained EEG recordings from 7 seizures. The EEG recording for each seizure started 60 seconds before the seizure onset and stopped 60 seconds after the seizure end. The median duration of the recordings was 295 seconds (range $238-465$ seconds). We refer to the period before, during and after the seizure as preictal, ictal and postictal, respectively. The recording channels from which the first signs of ictal activity and early propagation were recorded, the so-called seizure onset zone (SOZ), as well as the exact time of the beginning and end of the seizure were determined by a board-certified neurophysiologist (Rodrigo Rocamora). The data was recorded at $500 \mathrm{~Hz}$, and we applied a high-pass filter with a cutoff frequency of $0.5 \mathrm{~Hz}$ and a low-pass filter at $40 \mathrm{~Hz}$ using fourth-order Butterworth filters. We discarded a total of 16 channels because they were located in the white matter of the brain. Using all 40 remaining channels we made a bipolar reference between contacts that were neighboring at the same electrodes, resulting in $M=35$ signals. We down-sampled the signals by a factor of two to a frequency of $250 \mathrm{~Hz}$. The signals were analyzed using a moving windows technique with a window length of 16 seconds and an increment of one second between subsequent windows (93.75\% overlap). The total number of windows $Z$ depended on the length of the EEG recording, which in turn depended on the duration of the corresponding seizure.

For each seizure, we computed $L$ for all the pairs of signals and all windows with index $z=1, \ldots, Z$ leading to a connectivity matrix $W^{z}$ for each window. As stated above, the windows belong either to the preictal, ictal or postictal period. As the first step of evaluation, we averaged separately for each period resulting in $\langle W\rangle_{\text {period. }}$. In these averages, we discarded all the windows that included either the beginning or the end of the seizure and therefore contained activity from two distinct periods.

Recall that in the analysis of the model systems presented above we used a threshold $\eta$ to convert our continuous-valued result matrix $W$ to a binary adjacency matrix $R^{\eta}$. In this analysis of real-world data, we aim not only at detecting the existence, but also to estimate the strength of the directed connection between nodes. Accordingly, in Fig. 10 we display continuous-valued matrices $\langle W\rangle_{\text {period }}$ of the temporal means obtained separately for each period of one exemplary seizure. Values below the mean plus one standard deviation taken separately across the elements of the matrix for the three periods are thresholded and set to zero. The reconstructed matrix $\langle W\rangle_{\text {preictal }}$ [Fig. 10(a)] shows connections mainly for contacts that are spatially close. We also infer connections between signals recorded from TB, PH and other regions. Moreover, we also observe connections within the SOZ sampled by contacts in $\mathrm{AH}$ and $\mathrm{PH}$. In the ic- 

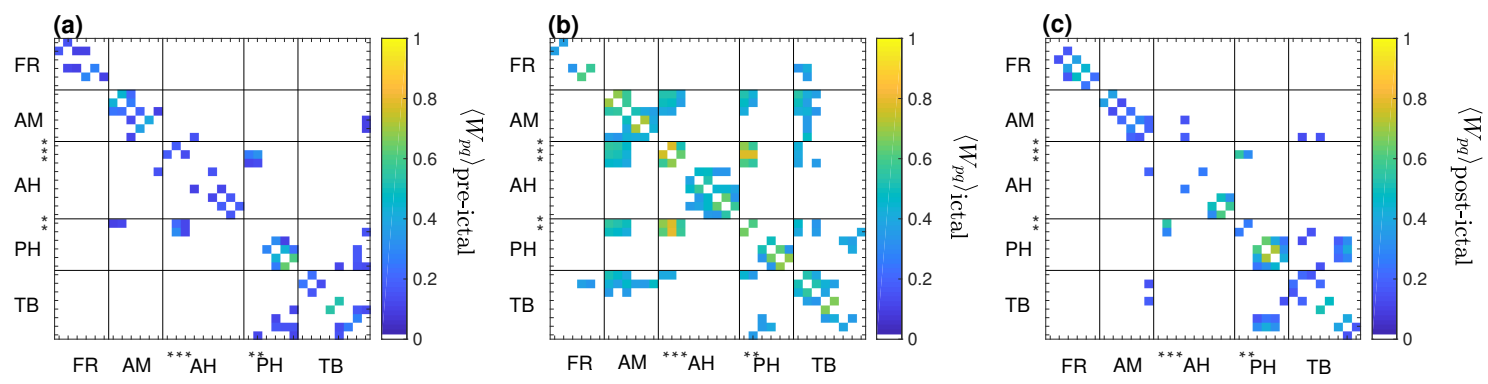

FIG. 10. High variability of connectivity structure is found across the different periods included in the EEG recording from an exemplary seizure. Temporal averages $\langle W\rangle_{\text {period }}$ for the third seizure for the periods preictal (a), ictal (b) and postictal (c). Values below the mean plus one standard deviation taken separately across the elements of the matrix for three periods are displayed in white. Black lines delimit the different regions of the brain where the electrodes were placed. Contact names placed in the SOZ are marked with an asterisk.

tal period [Fig.10(b)], the reconstructed matrix $\langle W\rangle_{\text {ictal }}$ shows an overall stronger interconnectivity between brain regions as compared to $\langle W\rangle_{\text {preictal }}$. Furthermore, some of the strongest connections are found for contacts placed in the SOZ. In contrast, the frontal region remains less connected as assessed by our inference results. This is in agreement with the clinical information that the seizure did not spread to this brain area. Finally, for $\langle W\rangle_{\text {postictal }}$ [Fig. 10(c)], as in the preictal period, most of the connections are found for spatially close contacts. However, in the postictal period, we infer stronger overall connectivity and more connections between brain regions. These results are not a special case for the particular seizure used as an example, and we found similar patterns regarding the structure of the reconstruction for all the other seizures. This consistency of the connectivity patterns across seizures is in agreement with the consistency of relative activation patterns that it was found for the same patient in [41].

In Fig. 10 we looked at the spatial structure of the connections by making a temporal average across the windows of the same period. In Fig. 11(a), instead, we look at the temporal evolution of the connectivity by making an average across all contacts within the same window $\bar{W}^{z}=\frac{1}{N(N-1)} \sum_{p, q ; p \neq q}^{M} W_{p, q}^{z}$. For all the seizures, we see that during the preictal period, the overall interdependence of the system is low. When the seizure starts, the quantity $\bar{W}^{z}$ quickly grows. In particular, for seizures $2-6$, we see two phases during the ictal period. One with higher interdependence at the beginning of the seizure, and one with smaller interdependence afterwards. In the postictal period, the quantity $\bar{W}^{z}$ goes down but remains higher than in the preictal period.

We continue by looking at the temporal evolution of the connectivity but now using the directional information provided by the measure $L$. We separately average across the matrix elements corresponding to the $\mathrm{SOZ}$ driving the rest of the contacts $\left(\bar{W}_{S \rightarrow R}\right)$ on the one hand, and the matrix elements corresponding to the opposite direction on the other hand $\left(\bar{W}_{R \rightarrow S}\right)$. Contacts placed in the resected area but not in the SOZ are not included in any of the averages. The temporal profile of $\bar{W}_{S \rightarrow R}-\bar{W}_{R \rightarrow S}$ is shown in Fig. 11(b). For all seizures, during both the pre- and postictal periods the connectivity is almost symmetric, and therefore the differences $\bar{W}_{S \rightarrow R}-\bar{W}_{R \rightarrow S}$ remain small. For the ictal period, apart from some variability across seizures, one common pattern is consistent across all seizures. Shortly after the seizure onset, we find a prominent asymmetry in connectivity in the sense that the SOZ is driving the remaining areas.

\section{DISCUSSION}

The aim of this study was to infer directed networks using solely the information of the signals of the nodes. To do so, we have presented a reconstruction method based on the state space measure $L$. We showed that for a subrange of the coupling strength and the link density, we were able to perfectly infer directed networks. This finding provided us with evidence that $L$ can be an effective measure to evaluate directed connections from multivariate signals and could potentially be used in a wide variety of problems $[2,4,5,32]$. Other studies that addressed the same problem, typically dealt with small systems and particular topologies [20-24] or analyzed the resulting structural network properties [19]. Here, instead, we were able to infer directed networks without choosing specific topology configurations. We also showed that dynamical noise can be beneficial for the reconstruction. This was true in particular for strongly coupled systems where noise prevents the system to enter into a synchronous state. We furthermore studied the performance of the method for increased system sizes. We showed that the performance remains constant if we properly adjust the link density.

Although pairwise approaches are prone to mistakenly detect indirect spurious connections, $L$ is performing well for a wide range of parameters. This good performance is specially noteworthy for high link density and high 

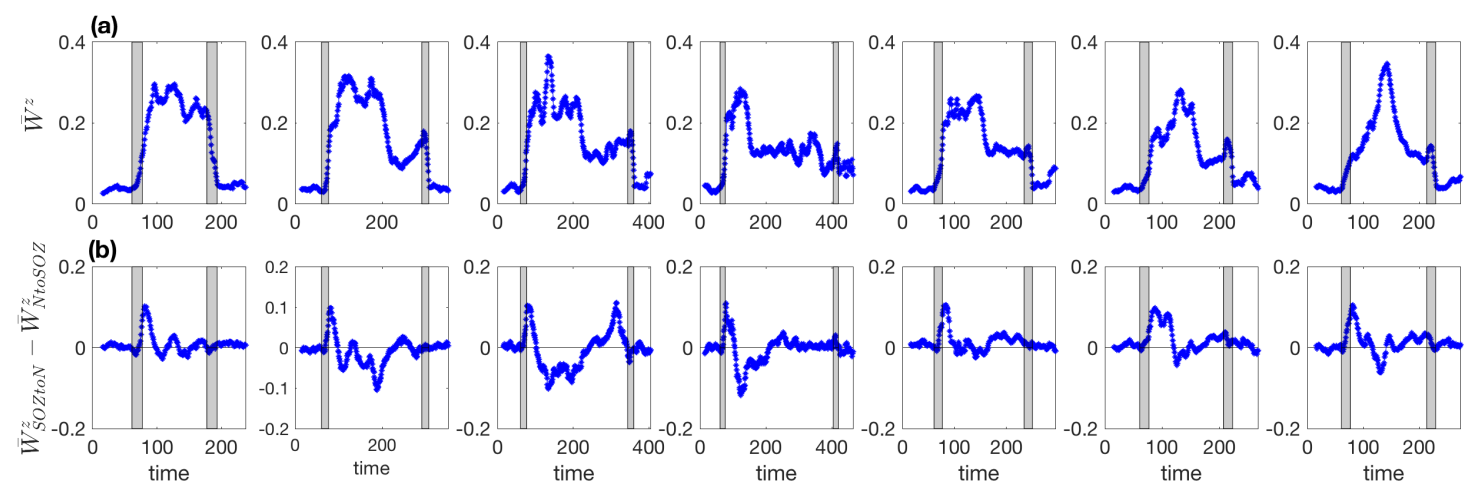

FIG. 11. A high overall connectivity is found during the seizure with a predominant direction from the SOZ to the remaining brain areas at the beginning of the seizure. (a) Spatially averaged connectivity matrix $\bar{W}^{z}$ in dependence on time for the 7 seizures. (b) Spatially mean connectivity of SOZ driving the rest of the contacts $\left(\bar{W}_{S \rightarrow R}\right)$ minus SOZ being driven $\left(\bar{W}_{R \rightarrow S}\right)$ by the rest of the contacts for the same 7 seizures. The grey frames depict windows that lay between two distinct periods. Left from the left frame is the preictal period, between the frames is the ictal period, and right from the right frame is the postictal period.

couplings at which the overall network dynamics become highly interdependent. This is because for such highly interdependent dynamics, the distinction between real links and indirect spurious links is difficult for any datadriven approach. To further improve the performance for highly interdependent dynamics, future work should aim at the development of multivariate extensions of the measure $L$, which are conditioned on subsets of the multivariate signals.

Applying our method to analyze EEG recordings from an epilepsy patient, we estimated the connectivity network for the three seizure periods. This resulted in three distinct structures corresponding to the three periods. We also observed that the strongest connections were the ones between contacts placed in the SOZ. We furthermore computed the temporal mean connectivity and noticed that it reflects the start and the end of the seizures. We also observed an asymmetrical pattern where the contacts situated in the SOZ were driving the rest of the contacts at the beginning of the seizure. This result is in agreement with other studies that reported driving of the SOZ [43-48]. In interpreting these results, we have to keep in mind that the available amount of data was limited, and a study of more patients, seizures and different anatomical locations of the SOZ should be carried out in order to make any strong claims. Nevertheless, these results show the potential of the method for this type of real-world data applications [33, 34].

In the first part of our study, we have used the area under the ROC curve to asses the performance of our reconstruction. This is a good way for testing the performance of our method in different settings when we know the ground truth. However, this procedure cannot be used when the exact topology it is not known, and a decision on how to threshold has to be made. Setting the threshold at a low (high) value, we will likely find false positives (negatives). A possible way to determine an optimal threshold is by plotting the ordered set as in Fig. 1. In this case, without the ground truth knowledge of the matrix A, we can of course not add color labels. Nonetheless, in case a clear gap exists in this ordered set [see Fig. 1(b)], a threshold can be readily drawn from within this gap. The setting of the threshold will have impact on the resulting network topology [49], and the choice of an optimal threshold without previous information of the system is still an open question. This is what we faced in the EEG example. However, despite not knowing the exact functional connectivity to validate our results, our findings are coherent with the medical information indicating that our method is extracting meaningful information about the system, and therefore it is a promising approach for medical applications.

To conclude, the MATLAB source codes used in this manuscript, including the calculation of $L$, will be available here [50].

\section{ACKNOWLEDGMENTS}

This work was funded by the EU via H2020 Marie SklodowskaCurie project COSMOS, grant no. 642563 (M.G.L., I.M., Z.L., and R.G.A). R.G.A. and C.G.B.M. acknowledge funding from the Spanish Ministry of Economy and Competitiveness (Grant FIS2014-54177-R) and the CERCA Programme of the Generalitat de Catalunya. C.G.B.M. acknowledges the support by the Spanish Ministry of Economy and Competitiveness under the Maria de Maeztu Units of Excellence Programme (MDM-20150502). Z.L. acknowledges funding from the Slovenian Research Agency via program Complex Networks P1-0383 and project J5-8236. 
[1] M. E. J. Newman, Networks, An Introduction (Oxford UP., 2007).

[2] D. Trejo Banos, A. J. Millar, and G. Sanguinetti, Bioinformatics 31(22), 3617-3624 (2015).

[3] D. Chicharro, R. G. Andrzejak, and A. Ledberg, BMC Neurosci. 12, P192 (2011).

[4] C. J. Oates, F. Dondelinger, N. Bayani, J. Korkola, J. W. Gray, and S. Mukherjee, Bioinformatics 30, i468 (2014).

[5] G. Tirabassi, L. Sommerlade, and C. Masoller, Chaos 27, 035815 (2017).

[6] M. Timme, Phys. Rev. Lett. 98, 224101 (2007).

[7] S. G. Shandilya and M. Timme, New J. Phys. 13, 13004 (2011).

[8] Z. Levnajić and A. Pikovsky, Phys. Rev. Lett. 107, 034101 (2011).

[9] Z. Levnajić and A. Pikovsky, Sci. Rep. 4, 5030 (2014).

[10] X. Han, Z. Shen, W.-X. Wang, and Z. Di, Phys. Rev. Lett. 114, 028701 (2015).

[11] E. S. C. Ching, P. Y. Lai, and C. Y. Leung, Phys. Rev. E 91, 030801(R) (2015).

[12] A. Pikovsky, Phys. Rev. E 93, 062313 (2016).

[13] R. Cestnik and M. Rosenblum, Phys. Rev. E 96, 012209 (2017).

[14] M. G. Leguia, R. G. Andrzejak, and Z. Levnajić, J. Phys. A 50, 334001 (2017).

[15] N. Rubido, A. C. Martí, E. Bianco-Martínez, C. Grebogi, M. S. Baptista, and C. Masoller, New J. Phys. 16, 093010 (2014).

[16] G. Tirabassi, R. Sevilla-Escoboza, J. M. Buldú, and C. Masoller, Sci. Rep. 5, 10829 (2015).

[17] E. Bianco-Martinez, N. Rubido, C. G. Antonopoulos, and M. S. Baptista, Chaos 26, 043102 (2016).

[18] D. Kugiumtzis, Phys. Rev. E 87, 062918 (2013).

[19] C. Koutlis and D. Kugiumtzis, Chaos 26, 093120 (2016).

[20] M. Winterhalder, B. Schelter, and J. Timmer, Chaos 17, 013110 (2007).

[21] B. Schelter, J. Timmer, and M. Eichler, J. Neurosci. Methods 179, 121 (2009).

[22] M. Jachan, K. Henschel, J. Nawrath, A. Schad, J. Timmer, and B. Schelter, Phys. Rev. E 80, 011138 (2009).

[23] L. Sommerlade, M. Eichler, M. Jachan, K. Henschel, J. Timmer, and B. Schelter, Phys. Rev. E 80, 051128 (2009).

[24] D. Kugiumtzis, Eur. Phys. J. Special Topics 222, 401 (2013).

[25] D. Chicharro and R. G. Andrzejak, Phys. Rev. E 80, 026217 (2009).

[26] S. J. Schiff, P. So, T. Chang, R. E. Burke, and T. Sauer, Phys. Rev. E 54, 6708 (1996).

[27] J. Arnhold, P. Grassberger, K. Lehnertz, and C. E. Elger, Physica D 134, 419 (1999).
[28] R. Q. Quiroga, J. Arnhold, and P. Grassberger, Phys. Rev. E 61, 5142 (2000).

[29] R. Q. Quiroga,A. Kraskov, T. Kreuz, and P. Grassberger, Phys. Rev. E 65, 041903 (2002).

[30] J. Serrà, X. Serra, and R. G. Andrzejak, New J. Phys. 11, 93017 (2009).

[31] R. G. Andrzejak and T. Kreuz, Europhys. Lett. 96, 50012 (2011).

[32] G. Sugihara, R. May, H. Ye, C. H. Hsieh, E. Deyle, M. Fogarty, and S. Munch, Science 338, 496 (2012).

[33] R. G. Andrzejak, D. Chicharro, K. Lehnertz, and F. Mormann, Phys. Rev. E 83, 046203 (2011).

[34] R. G. Andrzejak, K. Schindler, and C. Rummel, Phys. Rev. E 86, 046206 (2012).

[35] E. Pereda, R. Q. Quiroga, and J. Bhattacharya, Prog. Neurobiol. 77, 1 (2005).

[36] P. Laiou and R. G. Andrzejak, Phys. Rev. E 95, 012210 (2017).

[37] I. Malvestio, T. Kreuz, and R. G. Andrzejak, Phys. Rev. E 96, 022203 (2017).

[38] J. Theiler, Phys. Rev. A 34, 2427 (1986).

[39] A. Pikovsky, M. Rosenblum, and J. Kurths, Synchronization : a universal concept in nonlinear sciences (Cambridge University Press, 2001).

[40] D. A. Smirnov and R. G. Andrzejak, Phys. Rev. E 71, 036207 (2005).

[41] M. Vila-Vidal, A. Principe, M. Ley, G. Deco, A. Tauste Campo, and R. Rocamora, Clin. Neurophysiol. 128(6), 977 (2017) .

[42] A. Tauste Campo, A. Principe, M. Ley, R. Rocamora, and G. Deco, PLoS Bio. 16(4), e2002580 (2018).

[43] C. Flamm, A. Graef, S. Pirker, C. Baumgartner, and M. Deistler, J. Neurosci. Methods 214, 80 (2013).

[44] P. van Mierlo, E. Carrette, H. Hallez, R. Raedt, A. Meurs, S. Vandenberghe, D. Van Roost, P. Boon, S. Staelens, and K. Vonck, Epilepsia 54, 1409 (2013).

[45] A. Korzeniewska, M. Cervenka, C. Jouny, J. Perilla, J. Harezlak, G. Bergey, P. Franaszczuk, and N. Crone, NeuroImage 101, 96 (2014).

[46] C. M. Epstein, B. M. Adhikari, R. Gross, J. Willie, and M. Dhamala, Epilepsia 55, 2038 (2014).

[47] K. Lehnertz and H. Dickten, Phil. Trans. R. Soc. A 373, 2034 (2015).

[48] H. Dickten, S. Porz, C. E. Elger, and K. Lehnertz, Sci. Rep. 6, 34824 (2016).

[49] G. Cecchini, M. Thiel, B. Schelter, and L. Sommerlade, J. Neurosci. Methods 307, 31 (2018).

[50] http://ntsa.upf.edu/downloads 